\title{
The simulation research of electronic differential system for rear-wheel motor drive electric vehicle
}

\author{
Yanan $\mathrm{Gou}^{1, \mathrm{a}}, \mathrm{Xu} \mathrm{Tao}^{2, \mathrm{~b}}$ \\ ${ }^{1}$ College of Mechanical and Electrical Engineering; ${ }^{2}$ College of Information Science and \\ Engineering;Zaozhuang University Zaozhuang 277160, China

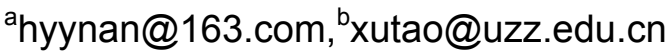

Keywords: rear-wheel drive, differential study, control theory, simulation research.

\begin{abstract}
The differential study of electronics for rear-wheel drive was studied recent years, the output speed is the system variables and the PID control strategy is the base, system strategy for rear-wheel drive was designed and differential system was simulated. Results show that through the calculation and adjustment of the speed, electronic differential control system can ensure the inside and outside vehicle wheel speeds keep basically constant in steering, so that the electronic differential for rear-wheel drive can be realized.
\end{abstract}

\section{Introduction}

The electric vehicle research is widely recognized and attended by the society from all walks of life with its growing advantages. The development road of electric vehicle in instead of traditional vehicles is more and more wide[1]. It is not only the change of power supply comparing with the traditional automobiles, electric drive automobiles become an important direction due to the various advantages. At present the study of rear-wheel electric drive has two aspects: one is the adaptive differential design, but the adaptive technology in the drive efficiency and the control strategies is limited because of its special motor design. Another one is rear-wheel drive differential design which uses differential control method[2,3]. The differential control system for the rear-wheel drive automobile is designed and the method is introduced.

\section{Differencial system structure}

From figure 1, the whole structure is shown and its strategy is mainly to control the differential drive when in turning. Hardware parts of the whole automobile include: the wheel in steering, accelerator and brake pedal, displacement sensors, the vehicle controller and motors. The total controller accepts all signals and automobile speed. The vehicle controller gets the distribution between angle in turning and each wheel speed according to the differential strategy, and then adjusts motor speed so as to control inside and outside wheel speed when steering.

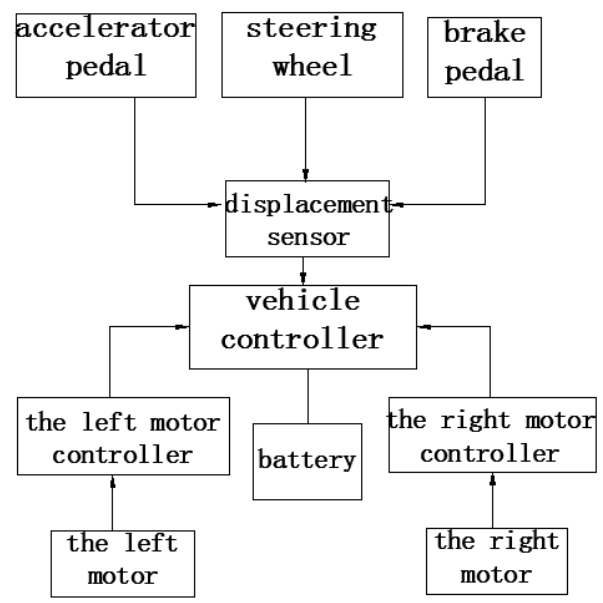

Fig. 1 Differencial system structure

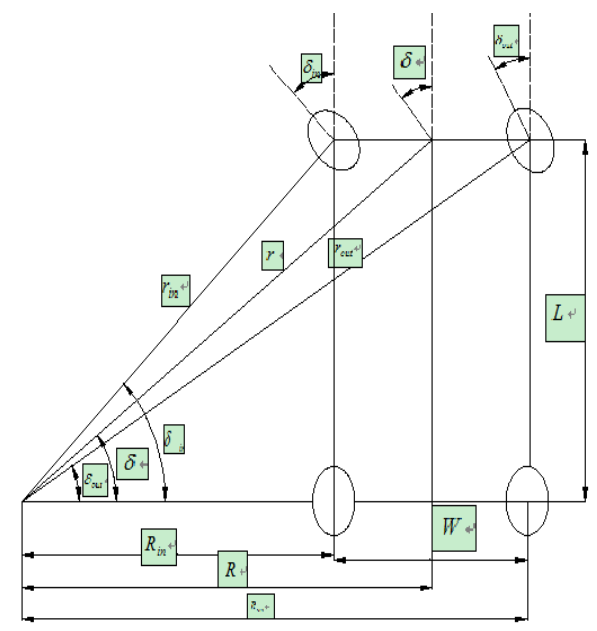

Fig. 2 Rear-wheel drive steering structure diagram 


\section{The electronic differential principle}

By reading references we know that differential is based on its theory, the vehicle controller controls the turning speed of the driving wheel so the wheel can obtaining corresponding rotational speed when in turning and then it can implement differential steering. The electric vehicles must ensure the wheel does not skid when driving in turning in the paper, and that is to say the wheels rolling distance is equal to the wheel heart parallel moving distance. The control strategy is that the instructions provided by steering wheel, accelerator pedal and the brake pedal are transmitted to the displacement sensor and are converted to analog signals, at last they are input to vehicle controller. Rotation speed that vehicle needs is transmitted to the controller to realize differential control.

3.1 The theoretical analysis

We can get the automobile steering structure diagram from figure 2 according to the Ackermann theory model and the geometrical relationship[4,5].

The steering angle $\delta$ is when the automobile in turining

$$
\tan \delta=\frac{L}{R}
$$

Among them: $\delta$ is the steering angle; $\boldsymbol{L}$ is the body length, $\boldsymbol{R}$ is the steering radius.

The two front wheel $\delta_{\text {in }}, \delta_{\text {out }}$ are

$$
\left\{\begin{array}{l}
\delta_{\text {in }}=\arctan \frac{L}{\frac{L}{\tan \delta}-\frac{W}{2}} \\
\delta_{\text {out }}=\arctan \frac{L}{\frac{L}{\tan \delta}+\frac{W}{2}}
\end{array}\right.
$$

Among them: $\boldsymbol{\delta}_{\text {in }}, \boldsymbol{\delta}_{\text {out }}$ are respectively the front axle inside and outside wheel steering angle; $\boldsymbol{W}$ is the body width.

The two front wheel speed $v_{\text {in }}, v_{\text {out }}$ are

$$
\left\{\begin{array}{l}
v_{\text {in }}=\frac{2 \pi R_{\text {in }}}{\Delta T}=\frac{2 \pi R-\pi W}{\Delta T} \\
v_{\text {out }}=\frac{2 \pi R_{\text {out }}}{\Delta T}=\frac{2 \pi R+\pi W}{\Delta T}
\end{array}\right.
$$

Among them: $\boldsymbol{v}_{\text {in }}, \boldsymbol{v}_{\text {out }}$ are respectively inside and outside speed of front wheel; $\boldsymbol{R}_{\text {in }}, \boldsymbol{R}_{\text {out }}$ are respectively corresponding steering radius; $\Delta \boldsymbol{T}$ is time rotating for a circle.

$$
\Delta T=\frac{2 \pi R}{v}
$$

Among them: $v$ is the motor speed. Put formula (4) into (3)

$$
\left\{\begin{array}{l}
v_{\text {in }}=v\left(1-\frac{W \tan \delta}{2 L}\right) \\
v_{\text {out }}=v\left(1+\frac{W \tan \delta}{2 L}\right)
\end{array}\right.
$$

Among them: $\boldsymbol{v}_{\text {in }}, \boldsymbol{v}_{\text {out }}$ are the variables related to the speed and the steering angle.

3.2 Electric vehicle dynamics model

From figure 3, we can get the wholer drive dynamics equation ignoring the suspension characteristics and considering the longitudinal velocity $v_{x}$ and lateral velocity $v_{y}$, the dynamics equation is

$$
\left\{\begin{array}{l}
m \dot{v}_{x}=\sum_{i=1}^{4} F_{x i}-m g \sin \alpha-\frac{1}{2} C_{D} A \rho v^{2} \\
m \dot{v}_{y}=\sum_{i=1}^{4} F_{y i} \\
I_{z} \dot{\gamma}=L_{f}\left(F_{y 1}+F_{y 2}\right)-L_{r}\left(F_{y 3}+F_{y 4}\right)+\frac{W}{2}\left(F_{x 1}+F_{x 3}\right)-\frac{W}{2}\left(F_{x 2}+F_{x 4}\right)
\end{array}\right.
$$




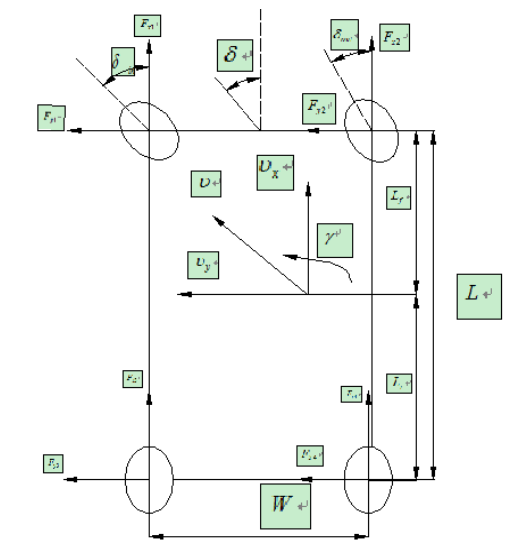

Fig. 3The dynamics diagram

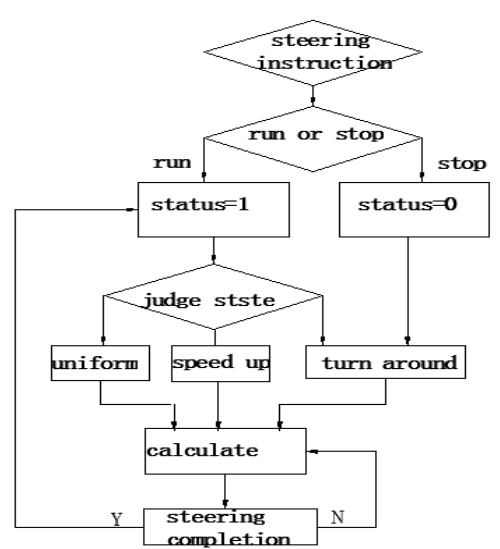

Fig. 4 Control method flow diagram

Among them: $\boldsymbol{m}$ is the whole quality; $\dot{v}_{x}, \dot{v}_{y}$ are the acceleration of the vertical and horizontal; $\boldsymbol{F}_{\boldsymbol{x} 1}, \boldsymbol{F}_{\boldsymbol{x} 2}, \boldsymbol{F}_{\boldsymbol{x} 3}, \boldsymbol{F}_{\boldsymbol{X} \mathbf{4}}$ are automobile longitudinal forces; $\boldsymbol{\alpha}$ is road slope; $\boldsymbol{F}_{\boldsymbol{y} 1}, \boldsymbol{F}_{\boldsymbol{y} \mathbf{2}}, \boldsymbol{F}_{\boldsymbol{y} \mathbf{3}}$, $\boldsymbol{F}_{\boldsymbol{y} 4}$ are automobile lateral forces; $\boldsymbol{C}_{\boldsymbol{D}}$ is resistance coefficient; $\boldsymbol{A}$ is windward area; $\boldsymbol{I}_{\boldsymbol{Z}}$ is rotary inertia around Z; $\boldsymbol{\gamma}$ is horizontal pendulum angular velocity; $\boldsymbol{L}_{\boldsymbol{f}}, \boldsymbol{L}_{\boldsymbol{r}}$ are respectively the length from the mass center to the front and rear axis wheel.

\section{The differential strategy}

The paper adopts independent double motor driving and they are on the two sides of the wheels which can form a certain speed difference when the wheel in turning. When $\delta>0$, the left side motor speed drops and the right is greater; when $\delta<0$, the right side motor speed drops and the results are in turn. We can get the control strategy flow diagram from figure 4 using PID method[6].

\section{The analysis results}

The differencial system is simulated and we can see the most parameters of the automobile model from table1.

Table 1 The model parameters

\begin{tabular}{cc}
\hline The parameter name & The parameter value \\
vehicle mass $(\mathrm{kg})$ & 1000 \\
driving wheel radius $(\mathrm{m})$ & 0.25 \\
rolling resistance coefficient & 0.016 \\
wind resistance coefficient & 0.34 \\
wheel base $(m)$ & 1.38 \\
shaft base $(m)$ & 2.345 \\
windward area $\left(\mathrm{m}^{2}\right)$ & 2 \\
the moment of inertia $\left(\mathrm{kg} \cdot \mathrm{m}^{2}\right)$ & 1650 \\
Height of mass center $(\mathrm{m})$ & 0.52
\end{tabular}

In this model, we assume the wheel is turning left and $\delta$ is on $\mathbf{2 0}^{\mathbf{0}}$, from figure 5 and figure 6 we can get vehicle horizontal pendulum angular velocity $\gamma$ and radius $\boldsymbol{R}$ under the differential control. From the pictures we can get that the two parameters reach steady state after a period of time. The speeds of the automobile increase from $0 \mathbf{k m} / \boldsymbol{h}$ to $60 \mathbf{~} \mathbf{m} / \boldsymbol{h}$ in the case of the $\delta$ unchanged, the curves of the two vehicle speeds and the curves of internal and external rotation speed ratio are as shown in figure 7 , figure 8 . We can get that the increase of two wheel rotational speeds basically keep pace with the increase of the vehicle speed, the internal and external rotation speed ratio basically remain unchanged. 


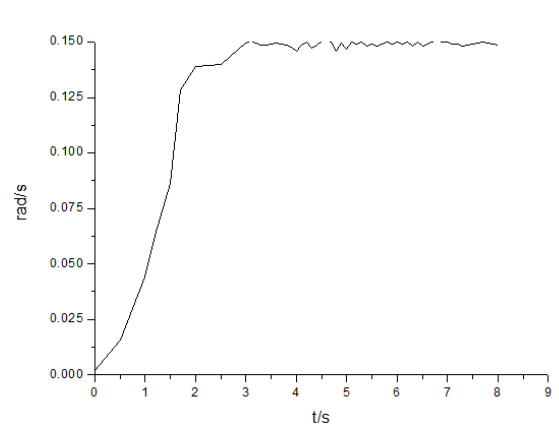

Fig.5 Horizontal pendulum angular velocity

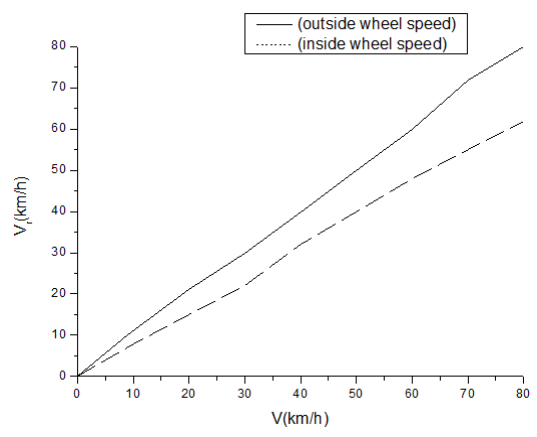

Fig.7 Inside and outside wheel speed

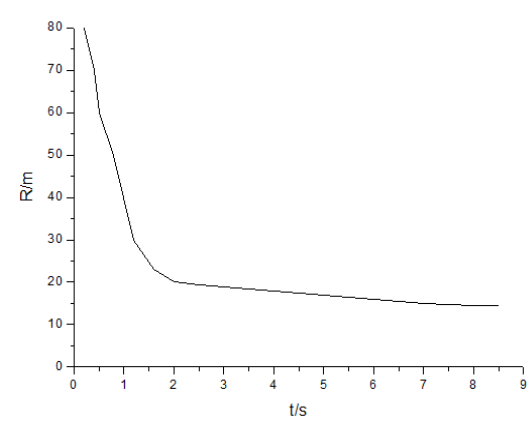

Fig. 6 Steering radius

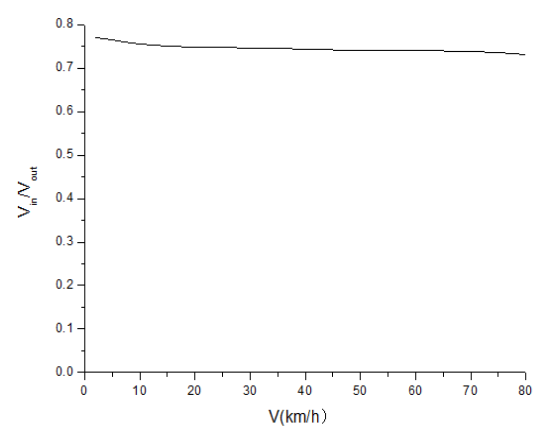

Fig. 8 Internal and external speed ratio

\section{Conclusion}

The differential method is studied and motor output speed is as control variable, we established differential system and put forward control method and simulated the model using MATLAB. The results prove through the calculation and adjustment of the speed, electronic differential control system can ensure the inside and outside vehicle wheel speeds keep basically constant in steering, that means it can realize electronic differential for rear-wheel drive.

\section{References}

[1] Xu Yin, Chen Dong. Summary on research of differential for a electric vehicle [J]. The Chinese mechanical engineering, 2011, 22(4): 498-503.

[2]Zhou Yong, Li Sheng jin, Tian Hai bo, Fang Zong de, Zhou Qi xun. Control method of electric differential of EV with four in-wheel motors [J]. Electric Machines and Control, 2007, 11(5): 467-471.

[3]Yan Yun bing, Peng Si lun. An analysis on the affecting factors of electric differential control for rear in-wheel motor drive vehicles [J]. Automotive Engineering, 2014, 36(2): 210-215.

[4] Jin Li qiang, Wang Qingnian, Zhang Yuanyuan. Experimental study on the differential capability of motorized wheels driving vehicles [J]. The Chinese mechanical engineering, 2007, 18(21): 2632-2636.

[5]Huang Bin, Wu Sen, Cao Zhengce. Investigation into Electronic differential technology for in-wheel motor drive electric vehicle [J]. Journal of Wu Han university of technology, 2013, 35(6): 134-138.

[6]Zhao Yane, Zhang Jian bin. Study on electronic differential control system of independent in-wheel motor drive electric vehicle [J]. Journal of system simulation, 2008, 20(18): 4767-4771. 